\title{
Intrinsic Viscosity of Polyelectrolytes in Salt Solutions*
}

\author{
Homei W. Chien, ${ }^{* *}$ Carl H. Isihara, ${ }^{* * *}$ and Akira Isihara \\ Statistical Physics Laboratory, \\ Department of Physics, State University of New York at Buffalo, \\ Buffalo, New York, 14214, USA.
}

(Received November 21, 1975)

\begin{abstract}
A theory is given for the inverse square-root dependence of the intrinsic viscosity of polyelectrolytes on the concentration of added salt. The results agree with the data by Noda, Tsuge, and Nagasawa. The existence of at least a partial drainage effect in polyelectrolytes is discussed.

KEY WORDS Polyelectrolytes / Intrinsic Viscosity / Concentration

Dependence /
\end{abstract}

The intrinsic viscosity has been a quantity of prime importance since the very beginning of polymer studies. In contrast with uncharged polymer molecules, the intrinsic viscosity of polyelectrolytes is even more interesting because of its sensitivity to added simple salts such as $\mathrm{NaCl}$ or $\mathrm{NaBr}$. This sensitivity must be crucial in biological activity. Moreover, the dependence on salt concentration gives rise to an extra dimension for testing theories.

Understanding the intrinsic viscosity of polyelectrolyte solutions requires greater theoretical efforts for the following reasons. First, the intrinsic viscosity has been shown experimentally ${ }^{1,2}$ to be proportional to $1 / c_{\mathrm{s}}{ }^{1 / 2}$, where $c_{\mathrm{s}}$ is the concentration of added salt, while most theories have predicted a $1 / c_{\mathrm{s}}$ dependence, ${ }^{3}$ except for those of Fixman ${ }^{4}$ and Yeh and Isihara. ${ }^{5}$ However, as has been pointed out by Noda, et al., ${ }^{2}$ Fixman's fundamental assumption that the concentration of counterions is much higher than $c_{\mathrm{s}}$ inside a polyion seems invalid. Yeh and Isihara assumed a segment distribution function referred to the molecular center of gravity. The distribution function vanishes at the origin and approaches the random-walk distribution function at large

* This work was supported by the National Science Foundation. It was submitted by one of the authors (H.W.C.) to the State University of New York at Buffalo for a partial fulfillment of the requirements for a $\mathrm{Ph}$. D. degree.

** Now at Emory University.

*** Now at Case-Western University. distances, and is of the type used by Bueche. ${ }^{6}$ Although Yeh and Isihara have reproduced the $c_{\mathrm{s}}^{-1 / 2}$ dependence, the assumed distribution function requires a better theoretical justification. Second, while the Stockmayer and Fixman relation ${ }^{7}$ seems to hold for the molecular weight dependence of the intrinsic viscosity, it is difficult to understand the curve with a negative slope which Noda, et al., recently found. Third, the same experimental data showed a significant deviation of the viscosity ratio $[\eta] /[\eta]_{0}$ plotted against $\left[M / c_{\mathrm{s}}\right]^{1 / 2}$ from Fixman's formula. There are still other somewhat minor theoretical difficulties. Motivated by these observations, we have written the present paper.

The concentration dependence of the intrinsic viscosity of polyelectrolytes can be interpreted qualitatively. Some of the charges of the polyion are neutralized by the salt ions so that as the concentration of salt increases the polymer shrinks, resulting in a decrease in the intrinsic viscosity.

We adopt a pearl-necklace model with $a$ for the diameter of the segment, considered to be spherical, and $b$ for the bond length. The molecular weight of a segment is denoted by $M_{0}$ and the total number of segments is $N$. The molecular weight of the polyelectrolyte is $M=N M_{0}$.

\section{VISCOSITY FORMULA}

An intrinsic viscosity formula which takes into consideration both the excluded volume 
effect and the hydrodynamic interactions between the segments has been given by one of us. ${ }^{8}$ In a simplified version based on a uniform expansion parameter it is ${ }^{9}$

$$
[\eta]=\frac{[\eta]_{0} \alpha^{2}}{1+a\left\langle R^{-1}\right\rangle}
$$

Here, $[\eta]_{0}$ is the intrinsic viscosity in the absence of the volume exclusion and the hydrodynamic interaction and $\alpha$ is the expansion factor for the square average end-to-end distance defined by

$$
\alpha=\frac{\left\langle R^{2}\right\rangle^{1 / 2}}{\left\langle R^{2}\right\rangle_{0}{ }^{1 / 2}}
$$

The denominator of eq 1 represents the hydrodynamic interaction effect. This is due to the finite size of the segments precisely as in the excluded volume effect. However, since the term "excluded volume effect" has been used only for expansion, we consider the hydrodynamic effect as separate from the excluded volume effect. by

The hydrodynamic interaction effect is given

$$
\left\langle R^{-1}\right\rangle=\left\langle R^{-1}\right\rangle_{0} \alpha^{-1}
$$

where $\left\langle R^{-1}\right\rangle_{0}$ represents the random-walk result:

$$
\left\langle R^{-1}\right\rangle_{0}=4 \pi \int w_{0}(R) R \mathrm{~d} R=\frac{1}{b}\left(\frac{6 N}{\pi}\right)^{1 / 2} \ln \left|\frac{2+3^{1 / 2}}{2-3^{1 / 2}}\right|
$$

$w_{0}(r)$ is the segment distribution function about the center of gravity for the case without exclusion volume. ${ }^{8}$ With eq 3 , the intrinsic viscosity given by eq 1 will be asymptotically proportional to $\alpha^{3}$ at high molecular weight.

Whatever the form of $w_{0}(R)$, the above average is correct in magnitude as far as random-walk processes are concerned. The average $\left\langle R^{-1}\right\rangle$ appears in eq 1 owing to the consideration of hydrodynamic interactions. In the past, it has been stated that the viscosity data of neutral polymers can be explained by a consideration of volume exclusion without hydrodynamic interaction. For polyelectrolytes, we keep this term and find its role.

Formula (1) is based on assigning a hydrodynamic force $\boldsymbol{F}(\boldsymbol{R})$ to every point $\boldsymbol{R}$ in space. This force is a function of a single variable $R$ and thus is different from the many-body force introduced by Kirkwood and Riseman. Because of this difference, the hydrodynamic interaction has been expressed essentially by the average $\left\langle R^{-1}\right\rangle_{0}$. Note here that we have used $\left\langle R^{-1}\right\rangle_{0}$ instead of $\left\langle R^{-1}\right\rangle$ in order to set aside the usual excluded volume effect.

The integral of eq (4) counts the number of segments in each "surface" element $4 \pi R \mathrm{~d} R$. However, this counting is not quite correct, because a single force $\boldsymbol{F}(\boldsymbol{R})$ represents the effects of a single segmental sphere at $\boldsymbol{R}$ and because the very volume exclusion of this segment does not allow other segments within its volume. That is, we should have counted the number of spheres instead of points. Moreover, a pair of neighboring segments cannot approach closer than the bond length $b$. It is important to take into consideration this smallest unit of distance because the effectiveness of the hard-sphere diameter $a$ is relative to $b$ and because the hydrodynamic interaction, being effectively proportional to $\left\langle R^{-1}\right\rangle$, is divergent at $R=0$. For these reasons, we correct the integration of eq 4 so as to collect $\pi(a / 2)^{2}$ per $\pi b^{2}$. Accordingly, we introduce a correction factor $(a / 2 b)^{2}$ into the hydrodynamic term $a\left\langle R^{-1}\right\rangle$ in the denominator of eq 1 .

The above correction is analogous to the quantum correction $h^{3}$ due to the Pauli principle to the classical phase space volume element $d p$ dr. It must be distinguished from the usual excluded volume effect, which is expressed by the factor $\alpha^{-1}$ in eq 3 . This factor represents the selfavoiding-walk effect on the random-walk processes and is independent of what we have just discussed, although the origin is the same. In fact, $\alpha$ depends on $N$ but the above correction is independent of $N$. The former correction is of long-range character and the latter is short-range. Note that $w_{0}(R)$ in eq 4 has been obtained for long distances and is not correct for short distances even for random walk processes.

We remark that the above correction merely changes the numerical values of $\alpha$ and $b$, which are only theoretical parameters. For the molecular weight dependence of $[\eta]$, the presence of $M$ in the denominator is actually crucial.

The hydrodynamic interaction term has been obtained by a volume integration of $1 / R$. Because this distance cannot also be arbitrary small, the combined factor $4 \pi R^{2} \mathrm{~d} R / R$ may be 
corrected. With this correction and somewhat more explicitly, eq 1 becomes

$$
[\eta]=\frac{[\eta]_{0} \alpha^{2}}{1+\frac{0.91}{\alpha}\left[\frac{a}{b}\right]^{3}\left[\frac{M}{M_{0}}\right]^{1 / 2}}
$$

in which the volume character of the hydrodynamic interaction is clearly manifested.

The ratio $[\eta] /[\eta]_{0}$ can be identified as the viscosity expansion coefficient $\alpha_{\eta}$ cubed, because the Einstein formula for the intrinsic viscosity is proportional to the volume occupied by a polymer molecule, and because there should be no hydrodynamic term when $\alpha=1$. The underlying assumption is that the polymer molecule is a solid sphere and no solvent molecule can go through the sphere nor even slip on the polymer surface.

In order to evaluate the intrinsic viscosity, one needs to evaluate the expansion parameter. For this evaluation, we assume that the interaction potential between a pair of segments consists of three parts: a hard-sphere potential, a screened Coulomb potential, and an attractive potential: i.e.

$$
\phi(r)=\left\{\begin{array}{l}
\infty, r<a \\
\frac{e^{* 2}}{\varepsilon_{\mathrm{D}} r} \exp \left(-\kappa(r-a)-\varepsilon_{2} \exp \left(-3 r^{2} / 2 \lambda^{2}\right),\right.
\end{array}\right.
$$

where $e^{*}$ is the effective charge on the segments, $\varepsilon_{\mathrm{D}}$ is the dielectric constant of the solution, and the attractive part of the potential is assumed to be given by an exponential function with two parameters. We have found that the combined total potential reproduces the usual molecular potential form rather well. Use of the Gaussian exponential function is just for convenience.

The screened Coulomb potential adopted here agrees with what the long-range limit of the pair distribution function of hard-sphere ions suggests. ${ }^{10}$ We use the result given by eq 13 of ref. 10:

$$
\Psi(r)=\frac{e^{* 2}}{\varepsilon_{\mathrm{D}} r} \frac{\exp (-\mu r)}{(1+8 \pi n \beta)^{2}}
$$

The screening constant $\mu$ is given by

$\mu=\kappa(1+8 \pi a n \beta)^{-1 / 2}$,

$\beta=1 / k T$ ( $n$ being the number of charges per unit volume) and

$$
\kappa^{2}=4 \pi n \beta \mathrm{e}^{2}
$$

where $e$ is the charge of simple ions in the solvent and $\kappa$ is the Debye screening constant for point ions. The factor $8 \pi a n \beta$ is small and is neglected in the above expression (7).

In the past, the screened potential for hardsphere ions has been determined by solving the Poisson-Boltzmann equation with the Debye screening constant. In this method, the hardsphere character of the ions is taken into consideration through a boundary condition on the surface of the sphere at the origin of the coordinate system. However, in the vicinity of the sphere, the Debye screening may not be used, since it is a quantity representing a longrange many-body effect. The potential in eq 7 has been determined from the asymptotic behavior of the pair distribution function, which is determined by the potential of the average force and is free from this drawback.

The Debye screening constant is determined by the ionic character of the solution and is related to the concentration of added salt. Assuming that the dielectric constant is of order unity within a polymer molecule and that the charge of the ions is $e$, the electronic charge, we have

$$
\begin{aligned}
\kappa^{2} & =8 \pi \mathrm{e}^{2} N_{\mathrm{a}} 10^{-3} c_{\mathrm{s}} / \varepsilon_{\mathrm{D}} / k T \\
& =9.004 \times 10^{16} c_{\mathrm{s}}
\end{aligned}
$$

where $c_{\mathrm{s}}$ is in $\mathrm{mol} / 100 \mathrm{ml}$.

We shall use the assumption that the screened Coulomb repulsion and the exponential attraction combined are small in comparison with the thermal energy so that a first-order calculation is possible. This does not mean that each of the two oppositely signed potentials is small.

The evaluation of $\alpha$ is then straightforward. We obtain

$$
\begin{aligned}
\alpha^{2}= & 1+\frac{4}{3} \xi \\
\xi= & \xi_{0} N^{1 / 2} \\
\xi_{0}= & \left(\frac{6}{\pi}\right)^{1 / 2}\left(\frac{a}{b}\right)^{3}\left\{1+\frac{3 e^{* 2}}{\varepsilon_{\mathrm{D}} k T}\left(\frac{1}{\kappa a}+\frac{1}{\kappa^{2} a^{2}}\right)\right. \\
& \left.-\frac{\varepsilon_{2}}{k T}\left[\left(\frac{\pi}{6}\right)^{1 / 2}\left(\frac{\lambda}{a}\right)^{3}-1\right]\right\}
\end{aligned}
$$

The expansion factor for the mean square 
radius of gyration is denoted by $r_{\mathrm{s}}{ }^{2}$. It is given by

$$
\begin{aligned}
\alpha_{\mathrm{s}}{ }^{2} & =1+\frac{134}{105} \xi \\
& =1+1.2763 \xi
\end{aligned}
$$

As mentioned before, the intrinsic viscosity is associated with $\alpha^{3}$. In order to find $\alpha^{3}$, Fixman used a device based on a differential equation with a uniform expansion of the bond length in proportion to $\alpha .{ }^{11}$ In order to derive his expression, we assume that the bond length associated with the unperturbed square average

$$
\left\langle r^{2}\right\rangle_{0}=N b_{0}{ }^{2}
$$

is characterized by a constant $b_{0}$, instead of $b$. In the presence of volume exclusion, the actual bond length $b$ which enters the expression of $\left\langle r^{2}\right\rangle$ together with $a$ may be replaced by $\alpha b_{0}$. Retaining only the factors outside of the curly brackets of eq 10 , we write

$$
\alpha^{2}=1+\frac{4}{3 \alpha} \bar{\xi}
$$

where $\bar{\xi}$ means $\xi$ with $b$ replaced by $b_{0}$. We assume that both $\bar{\xi}$ and changes in $\alpha$ are small so that, with neglect of a second-order small term, we have

$$
x \mathrm{~d} \alpha^{2}=\frac{4}{3} \mathrm{~d} \bar{\xi}
$$

Integrating this equation under the initial condition that $\alpha=1$ when $\bar{\xi}=0$, we find

$$
\alpha^{3}=1+2 \bar{\xi}
$$

For the expansion factor for the radius of gyration, the same procedure results in

$$
\begin{aligned}
\alpha_{\mathrm{s}}{ }^{3} & =1+\frac{3}{2} \times 1.276 \bar{\xi} \\
& =1+1.914 \bar{\xi}
\end{aligned}
$$

\section{RESULTS AND DISCUSSION}

The expansion parameter $\xi_{0}$ is associated with $c_{\mathrm{s}}$. When $c_{\mathrm{s}}$ is $0.01,1 / \kappa a$ is approximately 0.4 and its square is small. For higher concentrations, $1 /(\kappa a)^{2}$ is even smaller. The effective charge $e^{*}$ may be related to $e$ through an ionization factor $x$ as follows:

$$
e^{*}=x e
$$

Being an effective parameter, $x$ can depend on the concentration and the ionic strength of solutions. By comparing our viscosity formula with the data of Noda, et al, i.e., Table II of ref 2 , we have found that it varies slightly from 0.418 for $c_{\mathrm{s}}=0.01$ to 0.353 for $c_{\mathrm{s}}=0.5$. Since our viscosity formula includes several other parameters, we did not try to find best values for $x$ but rather have adopted $x=0.418$ for the following parameters:

$$
\begin{gathered}
a=8 \AA, \quad b=50 \AA, \quad \lambda=3.6 \AA \\
\varepsilon_{2}=(k / 10) \times 290, \quad N_{\mathrm{a}}=6.0229 \times 10^{23}, \quad T=290 \mathrm{~K}
\end{gathered}
$$

The diameter $a$ and the bond length $b$ correspond to a segment of our theoretical pearlnecklace model. Since the theory assumes flexibility, a segment corresponds to several monomer units. Since we are using a perturbation theory with $a / b$ as a convergence parameter, we have given a rather small ratio. The molecular weight of a segment has been assigned a convenient value of 100 .

With the above choice of the parameters, our theoretical relation between $\xi_{0}$ and $c_{\mathrm{s}}$ becomes

$$
\xi_{0}=-0.0129+\frac{0.051 x^{2}}{c_{\mathrm{s}}{ }^{1 / 2}}
$$

Hence, choosing $M_{0}{ }^{1 / 2}=10$ we obtain

$$
\alpha_{\eta}{ }^{3}=\frac{1+0.1276 \xi_{0} M^{1 / 2}}{1+\frac{3.7 \times 10^{-4}}{\left(1+0.1276 \xi_{0} M^{1 / 2}\right)^{1 / 2}}}
$$

We have illustrated $\alpha_{\eta}{ }^{3}$ in Figure 1. As we see, $\alpha_{\eta}{ }^{3}$ gives nearly a straight line when plotted against $M / c_{\mathrm{s}}{ }^{1 / 2}$. The degree of ionization is 0.103 and the viscosity constant $K=$ $1.02 \times 10^{-3}$ for PAA. Hence, $[\eta] / M^{1 / 2}$ in the plot in Figure 4 of Noda, et al., ${ }^{2}$ has been converted to $[\eta] /[\eta]_{0}$, by dividing the ordinate by 1.02 . The agreement between our theory and experiment is excellent. In particular, we note that even the case of $c_{\mathrm{s}}=0.5$, which has a negative slope, is in agreement. Such a negative slope has not been reproduced by the Stockmayer-Fixman theory.

The slopes in Figure 1 depend linearly on $c_{\mathrm{s}}{ }^{1 / 2}$, as is illustrated in Figure 2. According 


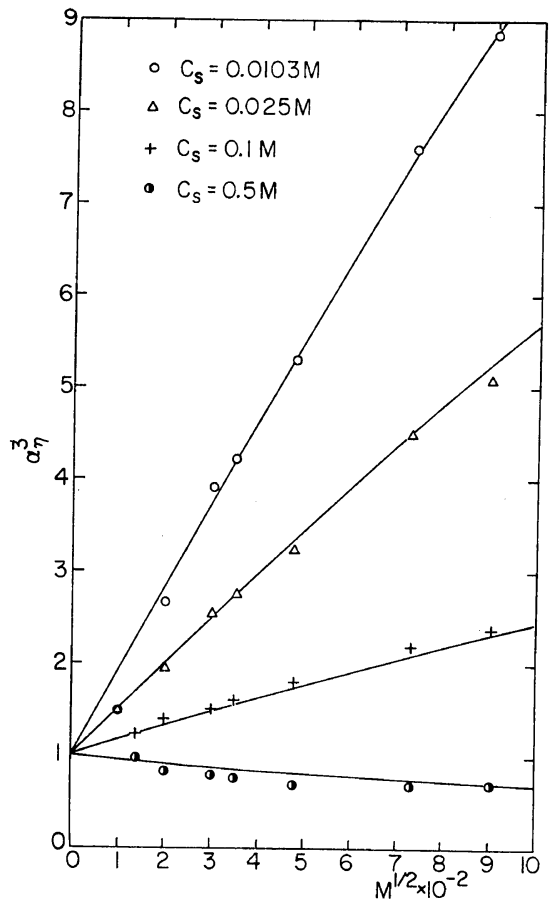

Figure 1. Viscosity ratio, $[\eta] /[\eta]_{0}=\alpha_{\eta}{ }^{3}$ plotted atainst $M^{1 / 2} \times 10^{-2}$ for four different concentrations of added salt. The data points represent PAA in NABr (Noda, Tsuge, and Nagasawa: ref. 2). The straight lines correspond to our theory.

to eq 17, the intercept is -0.0129 . This result can be compared with Figure 5 of Noda, et al., in which $B$ is plotted against $c_{\mathrm{s}}^{-1 / 2}$, where $B$ is the quantity appearing in the StockmayerFixman expression:

$$
[\eta] / M^{1 / 2}=K_{0}+0.51 \Phi_{0} B M^{1 / 2}
$$

The relation between $B$ and our $\xi_{0}$ is

$$
\frac{0.51\left(2.87 \times 10^{21}\right) 10^{-18}\left[B \times 10^{26}\right]}{\left(1.02 \times 10^{3}\right) \times 12.76}=\xi_{0}
$$

From Figure 5 of Noda, et al., we read

$$
B \times 10^{26}=-0.12
$$

Hence, the data by Noda, et al. give approximately the following experimental $\xi_{0}$ :

$$
\xi_{0 \exp }=0.0135
$$

in excellent agreement with our purely theoretical value -0.0129 from eq 17. As we mentioned before, $B$ depends actually on $i$, the

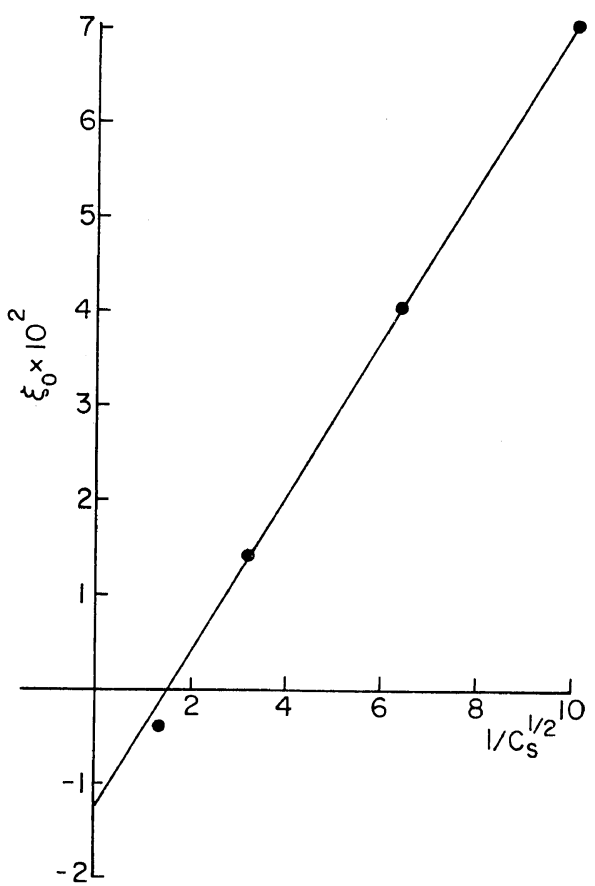

Figure 2. $\xi_{0}$ vs. $1 / c_{\mathrm{s}}{ }^{1 / 2} \cdot \xi_{0}$ is given by eq 17. Circles represent data by Noda, et al. $;^{2}$ the degree of ionization $i$ is 0.103 .

degree of ionization, so that we expect

$$
x=f\left(i, c_{\mathrm{s}}\right)
$$

By determining $x$ in successive approximations, we would be able to achieve even better agreement with the experimental results.

For polyelectrolytes, it is worthwhile to define

$\gamma=\frac{1.276}{M_{0}{ }^{1 / 2}}\left(\frac{6}{\pi}\right)^{1 / 2}\left(\frac{a}{b}\right)^{3} \frac{3 e^{* 2}}{\varepsilon_{\mathrm{D}} k T\left(\frac{8 \pi}{k T} \frac{e^{2}}{\varepsilon_{\mathrm{D}}} N_{\mathrm{a}} 10^{-3} a^{2}\right)^{1 / 2}}$

so that the electrostatic part of the expansion factor is

$$
\alpha_{\mathrm{se}}^{2}=1+\gamma M^{1 / 2} / c_{\mathrm{s}}{ }^{1 / 2}
$$

Numerically,

$$
\gamma=0.1276 \times 0.051 x^{2}
$$

For $x=0.418$ this yields

$$
\gamma=1.137 \times 10^{-3}
$$

Hence, the viscosity expansion associated with the electrostatic repulsion alone for high con- 


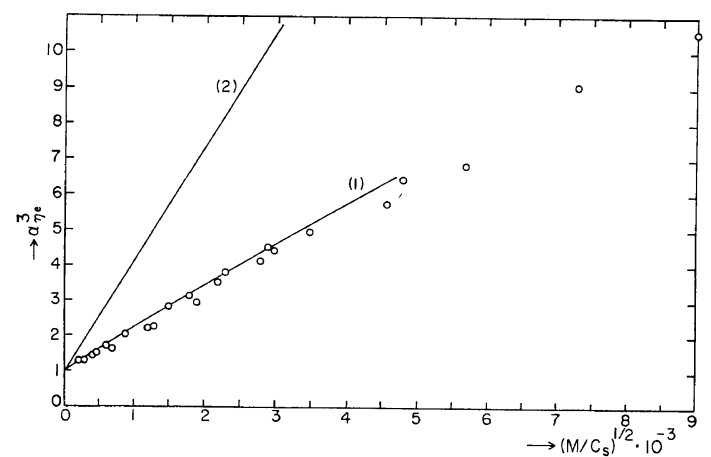

Figure 3. Electrostatic expansion factor $\alpha_{\eta \mathrm{e}}^{3} v s$. $\left(M / c_{\mathrm{s}}\right)^{1 / 2}$ : curve (1), our theory; circles data corresponding to those in Figure 1; curve (2), Fixman's relation.

centration is given by

$$
\begin{aligned}
\alpha_{\eta \mathrm{e}}^{3}= & {\left[1+1.137 \times 10^{-3}\left(M / c_{\mathrm{s}}\right)^{1 / 2}\right]\left[1+3.7 \times 10^{-4} M^{1 / 2}\right] } \\
& \times\left\{1+\frac{3.7 \times 10^{-4} M^{1 / 2}}{1+1.137 \times 10^{-3}\left(M / c_{\mathrm{s}}\right)^{1 / 2}}\right\}^{-1}
\end{aligned}
$$

This theoretical relation is plotted against $\left(M / c_{\mathrm{s}}\right)^{1 / 2} 10^{-3}$ in Figure 3. The extra $M$ dependence of our formula gives small fluctuations of the theoretical values for a given $\left(M / c_{\mathrm{s}}\right)$. However, these fluctuations are small and the solid Curve (1) represents our smoothed theoretical result, which has a slightly negative curvature.

We have explained the $1 / c_{\mathrm{s}}{ }^{1 / 2}$ dependence of the intrinsic viscosity of polyelectrolytes by neglecting the term which is proportional to $(\kappa a)^{-2}$. However, this depends on $\kappa$ and $a$. If the dielectric constant $\varepsilon_{\mathrm{D}}$ is of order 80 , the term $(\kappa a)^{-2}$ might become more important than $(\kappa a)^{-1}$. However, such a large value of $\kappa$ would decrease the $\gamma$ given by eq 23 significantly, requiring changes in some other parameters.

From our analysis in this paper, the hydrodynamical interaction term is important particularly in explaining the curve of a negative slope in Figure 1 which corresponds to $c_{\mathrm{s}}=0.5$ $M$. Therefore, it appears that there is at least a partial drainage effect in the polyelectrolyte solutions. Although further experimental tests are needed to be conclusive, it would be strange theoretically if there were no hydrodynamical interaction in any polymers, because the interaction is caused by volume exclusion just as in the case of the ordinary excluded volume effect.

The present work has been concerned with the concentration dependence of the viscosity ratio. In order to discuss the absolute magnitude of the intrinsic viscosity, we need to evaluate the viscosity at a reference state. We have also investigated the case of moderate to high salt concentration. In this rage, the limit in which the polymer charges are neutralized completely provides the reference state. For the opposite case of low concentrations, we need to develop a new theory. Our next task shall be the investigations of the low concentration case and also of the effect of the $(\kappa a)^{-2}$ term.

\section{REFERENCES}

1. A. Takahashi and M. Nagasawa, J. Amer. Chem. Soc., 86, 543 (1964); A. Takahashi, T. Kato, and M. Nagasawa, J. Phys. Chem., 71, 2001 (1967); N. Ohno, K. Nitta, S. Makino, and S. Sugai, J. Polym. Sci. Part C, 11, 413 (1973).

2. I. Noda, T. Tsuge, and M. Nagasawa, J. Phys. Chem., 74, 710 (1970).

3. A. Katchalsky and S. Lifson, J. Polym. Sci., 11, 409 (1953); O. B. Ptitsyn, Vysokomol. Soedin, 3, 1251 (1961); M. Kurata, J. Polym. Sci. Part C, 15, 347 (1966); Z. Alexandrowicz, J. Chem. Phys., 46, 3789, 3800 (1967); ibid., 474377 (1967); J. J. Hermans and J. T. G. Overbeek, Rec. Trav. Chim., 67, 761 (1948).

4. M. Fixman, J. Chem. Phys., 41, 3772 (1964).

5. R. Yeh and A. Isihara, J. Polym. Sci. Part A-2, 9, 373 (1971).

6. F. Bueche, J. Chem. Phys., 21, 208 (1953).

7. W. H. Stockmayer and M. Fixman, J. Polym. Sci. Part C, 1, 137 (1963).

8. A. Isihara, Advan. Polym. Sci., 5, 531 (1968).

9. R. Yeh and A. Isihara, J. Chem. Phys., 51, 1215 (1969).

10. A. Isihara and O. Aono, ibid., 48, 462 (1968).

11. M. Fixman, ibid., 36, 3132 (1962). 